\title{
Hands-on Training and International Experience in Engineering Education
}

\author{
Sanjeev Bedi \\ Professor Mechanical and \\ Mechatronics Engineering \\ Department \\ University of Waterloo, ONT \\ sbedi@mecheng1.uwaterloo.ca
}

\author{
Ajayinder Singh Jawanda \\ Assistant Professor Mechanical \\ Engineering Department \\ Thapar University, Patiala \\ asjawanda@thapar.edu
}

\author{
Ajay Batish \\ Professor Mechanical Engineering \\ Department \\ Thapar University, Patiala \\ abatish@thapar.edu
}

\begin{abstract}
The holistic training of an engineer includes hands-on experience and theoretical study. Engineering at the University of Waterloo (UW) is unique because of its co-op program, which exposes students to practice of engineering in industry, interwoven with academic terms in the university.

Five decades ago, when the university was founded, the students came from a demographic, that was split between a majority from rural and small urban centers. The students had some experience with tools and machines at high school and at home, as cars were simpler and home repair was a common activity. This was especially so on farms and in rural environments. This has gradually shifted to the present day when students primarily come from large urban centers and are very competent in working with hightech gadgetry like computers, communication devices and entertainment consoles. Conversely, nowadays most high school children are not exposed to working safely with tools and machines.

Over time co-op jobs have also shifted from hands-on engineering and shop floor work, to softer jobs using computers for design, analysis and managerial tasks. This has increased the challenge of engineering education where the facilities for teaching hands-on skills in the university are deterred by the lack of time in the curriculum, shortage of equipment and trained instructors. The economic environment of our nation has also evolved from a national manufacturing based economy a few decades ago, to a global economy of today. Industries have gone global in their routine functions, requiring engineers to routinely work in collaborative work with functionaries of their industry in other countries, with different culture, language and skill sets. It has thus become vital for engineering education to adapt and introduce the students to aspects of global engineering environment in the curriculum.
\end{abstract}

This paper details conduct of International Industrial Training Programs for the students of UW. These programs conducted over the past five years have addressed both issues of hands-on training and internationalization of engineering education. These programs provide skills and training on use of tools and machines culminating in a design and build project experience. This paper emphasizes safety and security in planning and implementing the programs in a foreign country. It also discusses issues related to international travel and cultural challenges faced by students and organisers. It summarizes the gains for the engineering students from the program based on immersive international exposure to a foreign culture through personal interactions with locals and planned trips, industrial practices, study of preparatory engineering courses like machining, welding, foundry, wood working, properties of materials and computer aided design leading to student designed and built projects.

\section{Introduction}

The decreasing hands-on shop work in School, College or University curriculums leads to producing an engineer lacking knowledge of working with their hands for product fabrication. The reduced importance of hands-on work in engineering education also has the potential of leading to very serious safety issues. The students have to work or manage production related engineering job for which they are presently less prepared than students a few decades back. Many industrial accidents can be attributed to human negligence or bad work design. Even students while pursuing their education need to be properly trained in the safe use of hand tools, power tools and machines. Neglecting the importance of this training could lead to grave accidents like the recent one at Yale University, USA [1].

Further, the curriculums of high school and 
Universities have had radical changes due to use of computing technology and escalating costs of maintaining machinery and skilled training staff to run shops for increasing number of students admitted to the engineering courses.

Economics has dictated that the manufacturing industry today is a global enterprise. Multinational corporations typically will have their functionaries in various parts of the world where the local conditions make the design, manufacturing, assembly and packaging reduce overall costs of launching products in the market. Engineers will typically have to deal with multicultural issues in routine besides travel to the subsidiaries spread across the world. A global outlook of an engineer has thus become desirable.

Both these issues have led to a need to relook at the engineering education system and come up with subtle changes in it to realise both these aims.

\subsection{Hands-on training in Engineering Education}

In the co-op based engineering program at Waterloo, we offer a strong theoretical grounding in various fundamental courses from sciences, humanities, general engineering courses which are interdisciplinary and the specialised engineering courses for the engineering program. In line with the bi-pronged learning paradigm of the co-op education, we rely on co-op work terms to provide practical "on the job" experience. Although it would be advantageous for our students to have practical "hands-on" skills, the lack of time in the curriculum and shortage of equipment, space and trained supervisors, are a significant deterrent. Increase in the class size to cater for a national demand of engineers for a growing population also puts pressure on the system to reduce the practical hands-on content for all engineering programs.

There are a number of fabrication projects included in the curriculum for hand-on work but the higher number of students to a project group means that the individual learning is focused on a few aspects.

At Waterloo there have been a number of student projects for design and fabrication competitions at national and international levels. Only a select few are exposed to the intense hands-on experience under these projects.

A few students get international exposure under the international student competitions for designs like the SAE F1 car, Hybrid car, Solar car or off-road vehicles. These international experiences are short and much specialised in their extent.

\subsection{Co-op program}

The University of Waterloo is known for its engineering education, at the heart of which is the differentiating factor of the co-op program. This interweaves the academic terms with practical experience in the Industry. The co-op programme offered by University of Waterloo provides the students a bi-pronged learning of the engineering discipline with majority of the teaching of 8 terms coming from the education programme within the university and the other 6 terms coming from practical training in industry.

University of Waterloo pioneered the co-op concept and has used it effectively to train some of the leading engineers in Canada. Over the years as the economy has taken a global flavour and manufacturing has shifted its base and the nature of coop jobs has taken an analytical flavour. UW students today graduate with reduced exposure to practical skills.

The quality of jobs offered to the student become more in engineering application as the student progresses to senior years of engineering. Even then only a small percentage of the co-op jobs demand hand-on work on product manufacture and fabrication. The shift in industry focus away from manufacturing has affected the co-op job content away from manufacturing and towards quality control, software and analysis, etc.

\subsection{Change in High school shops and hands-on work at home}

Engineers today have less exposure to engineering practice than a few decades ago. This can be attributed to changes in the Ontario school system, the job market and the economy.

The Ontario schools system went through an overhaul which has resulted in a reduction of practical, "hands on", content in courses. Furthermore, the changes have resulted in a concentrated program where students focus on obtaining good marks to get into university and sacrifice other activities such as practical training.

The economy has also changed from times when repair of automobiles was possible at home. Children brought up in rural environments were especially given work requiring use of hand tools and machinery from an early age. Communities were small and had to be independent in repair and maintenance of home appliances, automobiles and farm machinery. These skills honed up to high school were invaluable in producing engineers who were confident in working with their hands. This further helped them in industry which was largely manufacturing and demanded competence in manual work.

The modern manufacturing industry is largely 
automated but still requires the basic skills of working with hand tools and power tools. Engineering jobs as supervisors and managers also would require a competence in hands-on work for proper work design and safety. Loss of hands-on exposure early in growing stage also has an adverse effect on the number of people who become innovator hobbyists using basic hands-on engineering skills.

\section{Prevalent International exposure in Engineering Education}

The most profound change in society, namely, the shift to a global economy has created another dimension for engineering educators to address. To train our students at UW for the changing world, we currently have exchange programs with 50 other foreign institutions. The international experience from these programs suggests that the students amass learning beyond what would be possible in an institutional setting. These experiences are limited to a few of the students who apply for the few seats available in a foreign institution.

There are a number of institutions around the world which offer students international exposure. Recognizing a growing demand for internationallyexperienced engineering graduates, a group of leading universities around the world established the Global E3 in 1995 [2][3]. Students are able to study abroad for a semester and receive academic credit toward their degree at the Institution where they are registered for their degree course. IEE Exchange Programs offered by the Cockrell School of Engineering, The University of Texas at Austin to its students are exchange semesters at 11 select institutions from 10 countries across the world on similar lines [4]. New Jersey Institute of Technology has exchange agreements with institutions in France, Germany, Italy, and Sweden [5]. Similar mutual student exchange programs are supported by the Tokyo Institute of Technology School of Engineering with select Institutions around the world for course study [6]. The Nanyang Polytechnic, Singapore offers internship and attachment to the polytechnic practical projects in a number of engineering fields [7].

All these programs have academic equivalent courses in host institutions around the world which the student would study along with the local students and get credit for course passed in their degree back in the home Institution. The number of students who can take these is limited and select. The complete expenses for travel and stay are borne by the student.

The need is to have a program which is catering to the need of providing hands-on training using the design and build approach. The students need to be given knowledge of all the aspects of manufacturing technologies, training on safe and skilled use of the machines and equipment to be used for design and manufacturing of the Project. It is also combined with the aim of providing international exposure to industrial and cultural issues. The International Industrial Training Program (IITP) by UW in collaboration with Thapar University, Patiala, India has done precisely that.

\section{The International Industrial Training Program [8][9]}

At University of Waterloo we have tried to address the dual issues of a global economy and reduced practical skills by starting the International Industrial Training Program. The goal of this program is to provide a 12 week intensive practical skills training in an international setting.

The industrial training program was developed collaboratively by the University of Waterloo and Thapar University, Patiala, India. The International Industrial Training Program is hosted in Thapar University, Patiala (TU) and completely funded by the University of Waterloo. The host institute has a wellequipped workshop dedicated to training of engineering students. The facilities in the workshop are for Machining [Figure 1], Fitting [Figure 2], Pattern Making, Moulding, Casting [Figure 3], Welding, Forging, Tin Smithy, Electroplating, Carpentry, etc. with a team of instructors and assistants for each shop.

The main component of this program is a design and build project in the end of the 12 weeks of training. This project, done over a period of 4 weeks at the end of the program, requires the students to design a machine using a CAD system and then fabricate it using the resources of the workshop. This project has been very successful and is the highlight of this program. The program provides our first work term students with practical skills that are an asset as an engineer and help in obtaining great co-op job experience.

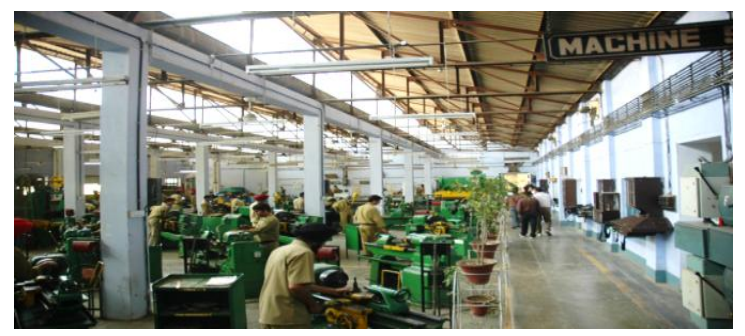

Figure1: Machine Shop 


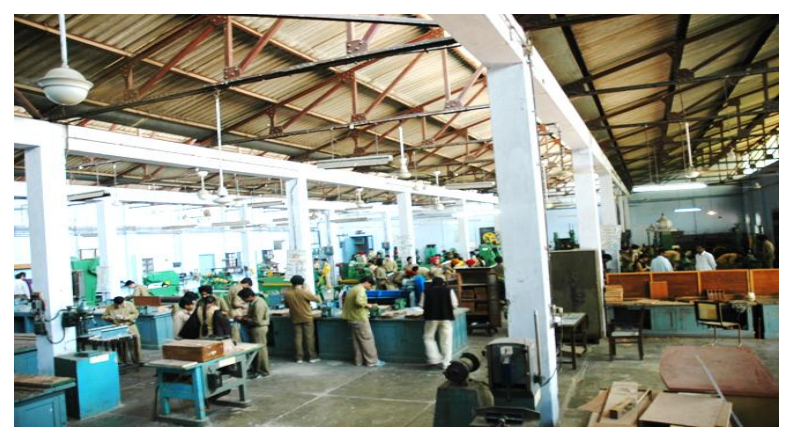

Figure 2: Sheet Metal, Fitting and Carpentry Shops

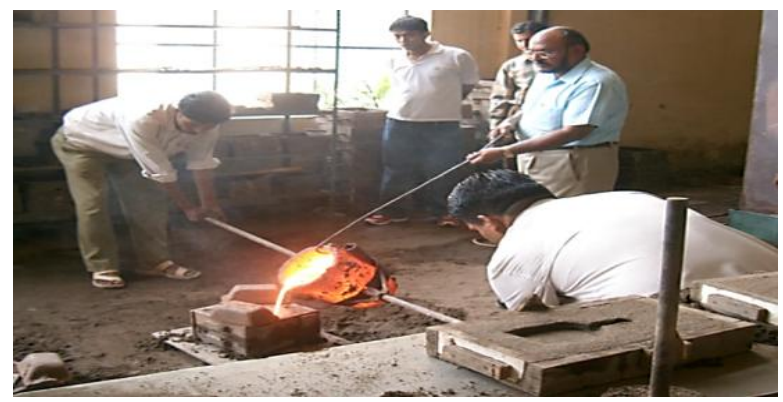

Figure 3: Casting Shop

The University has conducted five programs since spring 2005 in which it deputes 28 students to Thapar University, Patiala, India for the International Industrial Training Programme. The programme aims at providing the students training in mechanical engineering technologies such as machining, welding, $\mathrm{CAD}$, blue print reading, basic electricity, metrology and solid mechanics. The details of the curriculum, description of lectures and the practical content, list of tools and equipment to be used for the training, logistics of boarding, lodging and transportation, industrial and cultural trips and medical and security arrangements were all worked out in advance of every program.

A pilot International Industrial Training Program was launched in November of 2004 and since then has been offered to four more batches of about 28 students each.

\subsection{Conduct of the IITP}

There are two faculty coordinators one each from UW and TU. Their responsibilities are well defined in their domain and they serve as the communication link between the two institutions. The coordinators must be a responsible person with international or student experience.

The host coordinator ensures transport, lodging arrangements, food, help the students get over the culture shock and adjust to the environment, implement required safety training for various laboratory training activities and appropriate medical emergency procedure. Local site seeing trips, industrial trips and cultural events are planned in advance and organised on weekends. For the sake of efficiency there is a co-coordinator assisting the host coordinator for the conduct of the program at TU. This ensures a division of work in smooth conduct of courses and trips under the program and provides a standby coordinator in case of emergency.

Duties of the Waterloo coordinator are interviewing and selecting students for the program, briefing the students on host country specific issues, helping students prepare for the program (administration, visa, tickets etc.), liaising with host institute to ensure training goes on schedule, helping students with local issues, assisting students for the duration of the program, emergencies in Canada and abroad, debriefing upon return, overall management of the program.

The medical protocol is divided into three categories. Category one comprises of small injuries and minor ailments. Category two comprises of ailments requiring hospitalization and accidents. third category of medical protocol deals with severe injuries or ailments that are possibly life-threatening. The procedure to be adopted in case of these categories are detailed in advance and followed strictly.

TU ensures that all practices employed within the facilities where the students will be working comply with the Occupational Health and Safety (OHS) regulations that are required by the Government of India.

A time schedule for the entire duration of the program is made. This includes the detail schedule of Lectures, practice classes to teach the students the courses. The weekly allocated time in hours to lectures, practice and total hours for each course for the program are:

- Computer Aided Design \& Drafting 3, 3, 40

- Welding and Manufacturing 2, 6, 40

- Machining, Foundry and Pattern Shop 3, 8, 70

- Electrical \& Motor Control 2, 3, 20

- Strength of Materials 1, 2, 20

- Metrology 1, 2, 20

The students select a project in consultation with the course instructors. The project comprise of a design component and a fabrication component. The design must be conveyed as a CAD model. After the CAD model has been created, production drawing are generated and a prototype is built using the resources of the workshop in which the training is being imparted. The project concludes with a report and presentation. This report is submitted upon return to 
UW. The students can start on the project any time after the first 4 weeks but must finish before the program ends.

Projects that were built during this program include: Bio-Diesel Mixing Station; Acetylene Generator [Figure 4]; Cupola Furnace; Profile Gas Cutting Machine; Pallet Lifter [Figure 5]; Milling/Grinding Attachment for Lathe [Figure 6]; Hybrickshaw [Figure 7]; Air Compressor [Figure 8]; Wood Dust Vacuum System; to name a few.

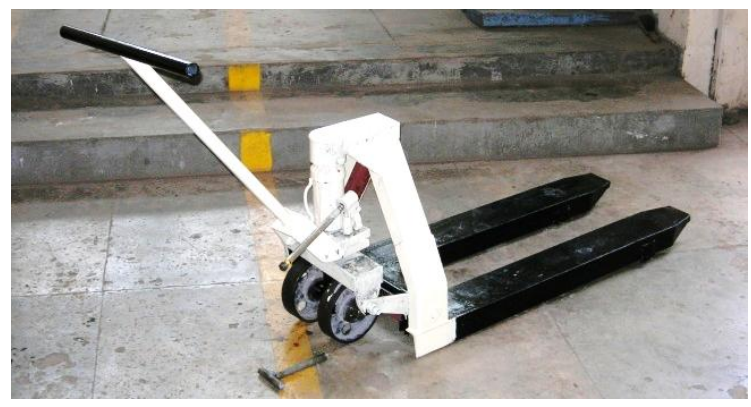

Figure 5: Pallet Lifter

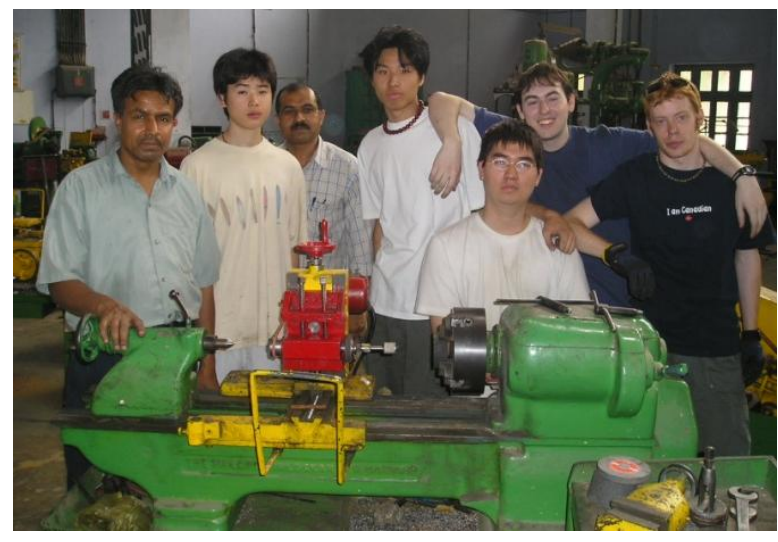

Figure 6: Milling/Grinding attachment for Lathe

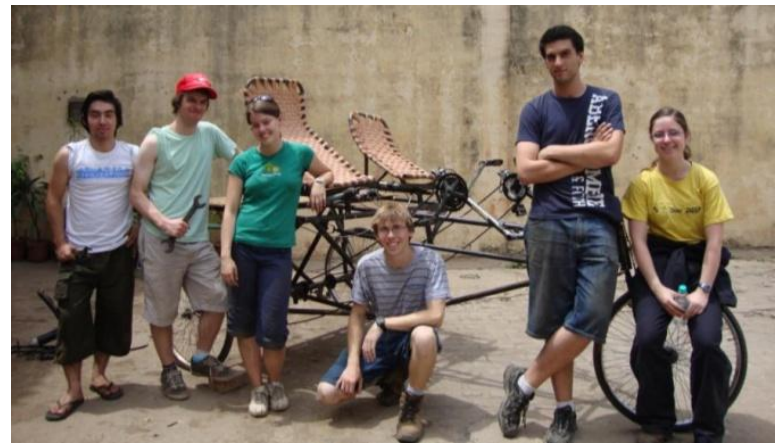

Figure 7: Hybrickshaw

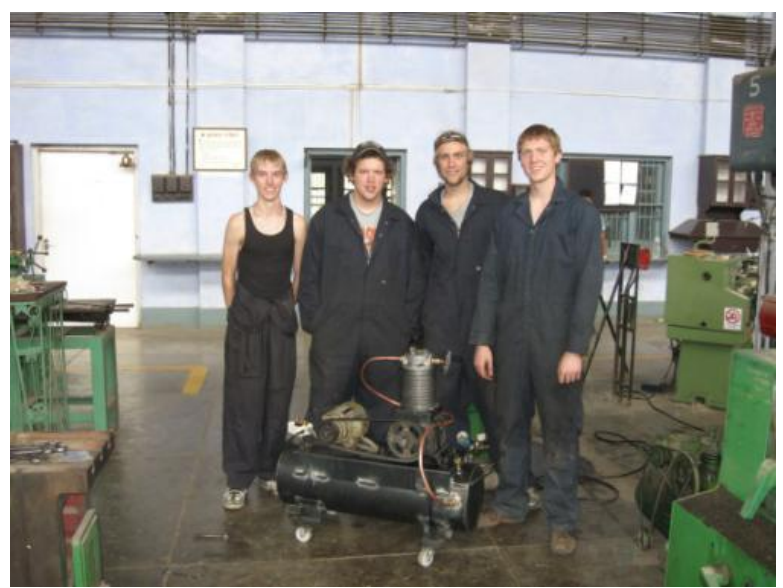

Figure 8: Air Compressor

\subsection{Cultural exposure: Trips, student interaction and activities, organised and self-initiated}

The trips to local industries to study the manufacturing culture of small to large scale manufacturing are planned and conducted by host institution. Trips to world renowned towns like New Delhi, Jaipur, Shimla, Chandigarh and world heritage sites like the Taj Mahal, Golden Temple are conducted as a part of the program as cultural exposure of the students.

As the UW students are housed along with the host student community in residences on campus, there is free interaction of the UW students with the local students. The students are encouraged and guided to take part in routine student activities at the host institution and local cultural festivals. Joint projects and collaborative events are encouraged and assistance provided to the UW students when asked for requirements difficult to meet in a foreign country with unfamiliar systems.

\subsection{Feedback and Evaluation}

The student assessment of the program is very good. Many have given feedback at their graduation and mentioned the IITP as the best co-op experience. The students especially like the design and build project. The international exposure and the trips were specially an eye opener for them.

The evaluation of the IITP is using written examinations for the theory courses, a practical examination of the CAD work along with the CAD modelling of the project and small job assignments for the different shops. Assessment by course instructors, lab instructor is done for the course work. Projects are assessed by the project mentors along with two the coordinators of the program based on the fabrication, final report and presentation by individual students. 
An individual student's qualitative evaluation is also sent by TU to UW. This gives feedback on the student's characteristics and the student's performance in the courses and project component of the IITP.

\section{Conclusion}

\subsection{Key Factors}

The most important ingredient in the program is the partnership with the host institute. In this program the cultivation of a good partnership with the host Institution is vital. The students in the pilot project liked the host institute and coordinators a lot and this is the key to the success of this program in subsequent years. In addition the host institute have a policy of imparting workshop training to students of all branches of engineering leading to a constant up gradation which is the basis of a good program on hands-on work training.

\subsection{Benefits}

The IITP training adds to the practical skills of our students and also provides them with learning from the international exposure making them valuable to co-op employers in future work terms and allow them to graduate with an enriched co-op experience.

The key benefits of the program are: practical skills training; international experience and networking.

- The training provides the students with an exposure to manufacturing processes. As part of the training program the students complete their first design and build project. This training and the project provide experience to the student that is valuable in obtaining of better jobs in subsequent terms. The training especially the project, also awakens an appreciation for manufacturing. Leading to a better engineer for future industrial work.

- Internationalization is recognized as an important element of education in light of the globalization of the world economy; however, universities are still fighting with ways to effectively include internationalization in their programs. IITP is a unique method of introducing internationalization in an engineering program.

- Due to the nature of coop our students have to pack up their bags every 4 months. This disruption stops strong networking from developing among classmates. The IITP puts the group of students together in a unique environment with goals. The special experiences in the program help in developing strong bonds within the group.
- Further benefits are in the introduction of jobs for the first work term students. This program takes 28 students away from the placement pool and trains them with better prospects for future jobs. This benefit has been reported by the students who had undergone the IITP.

\section{References}

[1] John Christoffersen, "Yale University physics student killed inside chemistry lab when lab machine snags hair" The Associated Press, The Canadian Press, http://ca.news.yahoo.com/yale-universitystudent-dies-apparent-accident-chemistry-lab20110413-071100-234.html Wed, 13 Apr, 2011.

[2] The Global Engineering Education Exchange Program -Global E3, http://www.iie.org/programs/GlobalE3/.

[3] L. A. Gerhardt and S.Martin The Global Engineering Education Exchange Program - A Worldwide Initiative, 29th ASEE/IEEE Frontiers in Education Conference, November, 1999 San Juan, Puerto Rico, pp10 - 13, http://fieconference.org/fie99/papers/1127.pdf.

[4] IEE Exchange Programs - Cockrell School of Engineering, The University of Texas at Austin, http://www.engr.utexas.edu/undergraduate/iee/6567iee-study-abroad-programs.

[5] New Jersey Institute of Technology, http://www.njit.edu/studyabroad/programs/exchangeprograms.php.

[6] Tokyo Institute of Technology School of Engineering, http://www.eng.titech.ac.jp/general/topics_e.html.

[7] Nanyang Polytechnic, Singapore, http://www.nyp.edu.sg/SEG/seg_attachment_foreign.h tml.

[8] International Industrial Training Program at University of Waterloo, ONT, Canada, http://www.itp.uwaterloo.ca/.

[9] IITP at Thapar University, Patiala, http://www.thapar.edu/pagemicro.asp? subId=15\&microId=57\&childId $=155$. 